\title{
Switched Networks and Complementarity
}

\author{
M. K. Çamlıbel, W. P. M. H. Heemels, A. J. van der Schaft, Fellow, IEEE, and J. M. Schumacher
}

\begin{abstract}
A modeling framework is proposed for circuits that are subject both to externally induced switches (time events) and to state events. The framework applies to switched networks with linear and piecewise-linear elements, including diodes. We show that the linear complementarity formulation, which already has proved effective for piecewise-linear networks, can be extended in a natural way to also cover switching circuits. To achieve this, we use a generalization of the linear complementarity problem known as the cone-complementarity problem. We show that the proposed framework is sound in the sense that existence and uniqueness of solutions is guaranteed under a passivity assumption. We prove that only first-order impulses occur and characterize all situations that give rise to a state jump; moreover, we provide rules that determine the jump. Finally, we show that within our framework, energy cannot increase as a result of a jump, and we derive a stability result from this.
\end{abstract}

Index Terms -Complementarity systems, hybrid systems, ideal diodes, ideal switches, piecewise-linear systems.

\section{INTRODUCTION}

$\mathbf{T}$ HE standard literature on dynamical systems is mostly concerned with systems that evolve in time according to a set of rules depending smoothly on the current state of the system. However, in electrical engineering as well as in other fields, one is often confronted with systems that are most easily modeled as going through a succession of periods of smooth evolution separated by instantaneous events that mark transitions of one set of laws of evolution to another. Events may be externally induced (as in the case of switches) or internally induced (as in the case of diodes). To come up with a precise mathematical formulation of systems with events is a nontrivial matter, in particular because one has, in general, to allow for the possibility that a state jump is associated with events and so it would be too restrictive to require solutions to be continuous, let alone differentiable.

Manuscript received September 9, 2002; revised April 14, 2003. This work was supported by the Eurepean Union Project SICONOS under Grant IST-200137172 and by STW under Grant EES 5173. This paper was recommended by Guest Editor M. di Bernardo.

M. K. Çamlıbel is with the Department. of Electronics Engineering, Dogus University, 34722 Istanbul, Turkey, the Department of Econometrics and Operations Research, Tilburg University, 5000 LE Tilburg, The Netherlands, and also with the Department of Electrical Engineering, Eindhoven University of Technology, 5600 MB Eindhoven, The Netherlands. (e-mail: kcamlibel@dogus.edu.tr).

W. P. M. H. Heemels is with the Department of Electrical Engineering, Eindhoven University of Technology, 5600 MB Eindhoven, The Netherlands (e-mail: w.m.p.h.heemels@tue.nl).

A. J. van der Schaft is with the Faculty of Mathematical Sciences, University of Twente, 7500 AE Enschede, The Netherlands (e-mail: twarjan@math.utwente.nl).

J. M. Schumacher is with the Department of Econometrics and Operations Research, Tilburg University, 5000 LE Tilburg, The Netherlands (e-mail: jms@uvt.nl).

Digital Object Identifier 10.1109/TCSI.2003.815195
It is the main purpose of this paper to propose a modeling framework for systems with events, designed in particular, for switched piecewise-linear networks. Our approach is based on the complementarity modeling that was used in [15] for dynamic networks with diodes. Here, we extend the framework of [15] to include also external switches. It turns out that the extension can be carried out in a very natural way. Instead of working with the cone of elementwise nonnegative vectors as in [15], we use here cones of a more general type. This corresponds to the generalization of the linear complementarity problem (LCP) of mathematical programming to a "cone-complementarity problem" (cf. for instance [10, p. 31]). This generalization brings a more geometric flavor to the setting of [15] and may be useful as well in the modeling of mode-switching elements other than diodes. Essentially, we describe switched piecewise-linear networks as cone-complementarity systems that are switched in time, from between several different cones from a given family. In addition to the notion of cone complementarity, the concept of passivity is central to the development of this paper; in fact, our main results all assume passivity.

As already noted, one of the main problems in setting up a rigorous framework for switched systems is to take into account the possibility of state jumps. We need a sufficiently rich solution space that allows discontinuities in state trajectories, and, consequently even impulses in input trajectories. In this paper, we choose a distributional framework. Although this choice effectively limits us to considering only (piecewise) linear networks, an advantage of using distributions is that we do not need to impose a priori a restriction on the nature of the jumps; rather we can prove that only first-order impulses arise, even though our setting in principle allows distributional solutions of arbitrarily high order.

\section{NotATION AND PRELIMINARIES}

The following notational conventions will be in force.

For any set $S, 2^{S}$ denotes the power set, i.e., the set of all subsets of $S$. The $n$ tuples of elements of $S$ will be denoted by $S^{n}$ as usual. The set of real numbers is denoted by $\mathbb{R}$. $\mathbb{R}_{+}$ stands for the set of nonnegative real numbers, i.e., $\{\rho \in \mathbb{R} \mid$ $\rho \geqslant 0\} . \mathbb{C}$ denotes the set of complex numbers. For a complex number $z, \operatorname{Re} z$ stands for the real part. The notations $v^{T}$ and $v^{*}$ denote the transpose and conjugate transpose of a vector $v$. When two vectors $v$ and $w$ are orthogonal, i.e., $v^{T} w=0$, we write $v \perp w$. Inequalities for real vectors must be understood componentwise.

The notation $\mathbb{R}^{n \times m}$ denotes the set of $n \times m$ matrices with real elements. The transpose of $A$ is denoted by $A^{T}$. Let $A^{n \times m}$ be a matrix. We write $A_{i j}$ for the $(i, j)$ th element 
of $A$. For $J \subseteq\{1,2, \ldots, n\}$, and $K \subseteq\{1,2, \ldots, m\}, A_{J K}$ denotes the submatrix $\left\{A_{j k}\right\}_{j \in J, k \in K}$. If $J=\{1,2, \ldots, n\}$ $(K=\{1,2, \ldots, m\})$, we also write $A_{\bullet} K\left(A_{J_{\bullet}}\right)$. If $n=m$ and $J=K$, the submatrix $A_{J J}$ is called a principal matrix of $A$ and the determinant of $A_{J K}$ is called a principal minor of $A$. In order to avoid bulky notation, we use $A_{J K}^{T}$ and $A_{J J}^{-1}$ instead of $\left(A^{T}\right)_{J K}$ and $\left(A_{J J}\right)^{-1}$, respectively.

Let $M$ be the square matrix. As usual, we say that $M$ is symmetric if $M=M^{T}$ and skew-symmetric if $M=-M^{T}$. The matrix $M$ (not necessarily symmetric) is said to be nonnegative definite if $v^{T} M v \geqslant 0$ for all vectors $v$. We say that $M$ is positive definite if $M$ is nonnegative definite and $v^{T} M v=0 \mathrm{im}$ plies $v=0$. Sometimes, we write $M \geqslant 0(M>0)$ by meaning that $M$ is nonnegative (positive) definite. In the obvious way, we define nonpositive definite and negative definite matrices. For two matrices $M$ and $N$ with the same number of columns, $\operatorname{col}(M, N)$ will denote the matrix obtained by stacking $M$ over $N$. The identity matrix will be denoted by $I$, the zero matrix by 0 .

A triple of matrices $(A, B, C) \in \mathbb{R}^{n \times n} \times \mathbb{R}^{n \times m} \times \mathbb{R}^{m \times n}$ is said to be minimal if $\operatorname{rank}\left(B, A B, \ldots, A^{n-1} B\right)=n$ and $\operatorname{rank}\left(\operatorname{col}\left(C, C A, \ldots, C A^{n-1}\right)\right)=n$.

A rational matrix $G(s)$ is said to be proper if $\lim _{s \uparrow \infty} G(s)$ is well-defined and finite. It is said to be strictly proper if it is proper and the above-mentioned limit is zero.

A subset of $\mathbb{R}^{n}$ is said to be polyhedral if it is of the form $\left\{v \in \mathbb{R}^{n} \mid A v \leqslant b\right\}$ for some matrix $A$ and a vector $b$.

Let $f$ be a function. We write $\left.f\right|_{\Omega}$ for the restriction of $f$ to the set $\Omega$. The notation $f(\tau+)(f(\tau-))$ will denote the limit $\lim _{t \uparrow \tau} f(t)\left(\lim _{t \downarrow \tau \tau} f(t)\right)$ whenever it is well defined.

The set of all Lebesgue measurable, square integrable functions $f: \Omega \leftarrow \mathbb{R}$ will be denoted $\mathcal{L}_{2}(\Omega)$. In case, $\Omega=\mathbb{R}_{+}$, we write only $\mathcal{L}_{2}$. The notation $\mathcal{L}_{2}^{\text {loc }}$ denotes locally $\mathcal{L}_{2}$ functions, i.e., the set $\left\{f|f|_{t, T} \in \mathcal{L}_{2}\right.$ for all $\left.0 \leqslant t<T\right\}$.

Dirac distribution supported at $\theta$ will be denoted by $\delta_{\theta}$ and its $k$ th derivative by $\delta_{\theta}^{(k)}$. When it is supported at zero, we usually write $\delta$ and $\delta^{(k)}$.

We say that a proposition $\mathcal{P}(\rho)$ holds for all sufficiently small (large) $\rho$ if there exists $\rho^{\prime}$ such that $\mathcal{P}(\rho)$ holds for all $0<\rho \leqslant \rho^{\prime}$ $\left(\rho^{\prime} \leqslant \rho\right)$.

\section{A. Cones and Dual Cones}

Definition II.1: A set $\mathcal{C} \subseteq \mathbb{R}^{\ell}$ is said to be a cone if $v \in \mathcal{C}$ implies that $\alpha v \in \mathcal{C}$ for all $\alpha \geqslant 0$.

Definition II.2: For any nonempty set $\mathcal{Q} \subseteq \mathbb{R}^{\ell}$, we define the dual cone as the set $\left\{w \in \mathbb{R}^{\ell} \mid w^{T} v \geqslant 0\right.$ for all $\left.v \in \mathcal{Q}\right\}$. It will be denoted by $\mathcal{Q}^{*}$.

Note that the dual cone of a set can be defined even if the set is not a cone. It is immediate that a dual cone is always closed and convex.

\section{B. Complementarity Problems}

The LCP plays quite an important role in the sequel. In what follows, we will quote some well-known facts from complementarity theory.
Definition II.3: $\operatorname{LCP}(q, M)$ : Given a vector $q \in \mathbb{R}^{m}$ and a matrix $M \in \mathbb{R}^{m \times m}$ find a vector $z \in \mathbb{R}^{m}$ such that

$$
\begin{aligned}
z \geqslant 0 \quad q+M z & \geqslant 0 \\
z^{T}(q+M z) & =0 .
\end{aligned}
$$

We say that the $\operatorname{LCP}(q, M)$ is solvable if such a $z$ exists. In this case, we also say that $z$ solves (is a solution) of $\operatorname{LCP}(q, M)$. The set of all solutions of $\operatorname{LCP}(q, M)$ is denoted by $\operatorname{SOL}(q, M)$. A weaker notion than solvability is feasibility. The $\operatorname{LCP}(q, M)$ is said to be feasible if there exists $z$ such that (1a) is satisfied.

Theorem II.4: The following statements hold.

1) $[10$, Th. 3.3.7]: $\operatorname{LCP}(q, M)$ has a unique solution for all $q \in \mathbb{R}^{m}$, if and only if all principal minors of $M$ are positive.

2) $[10$, Cor. 3.8.10, Th. 3.8.13]: Suppose that $M$ is nonnegative definite. Then, the following statements are equivalent:

a) $q \in(\mathrm{SOL}(0, M))^{*}$;

b) $\operatorname{LCP}(q, M)$ is feasible;

c) $\operatorname{LCP}(q, M)$ is solvable.

Remark II.5: Matrices all of whose principal minors are positive are known as $P$ matrices in complementarity theory (see, e.g., [10, Def. 3.3.1]). In particular, positive definite matrices are in this class.

One interesting generalization of the LCP can be obtained by modifying the conditions (1a) as follows.

Definition II.6: $\operatorname{LCP}_{\mathcal{C}}(q, M)$ : Given a cone $\mathcal{C}$, a vector $q \in$ $\mathbb{R}^{m}$, and a matrix $M \in \mathbb{R}^{m \times m}$ find a vector $z \in \mathbb{R}^{m}$ such that

$$
\begin{aligned}
z \in \mathcal{C} \quad q+M z & \in \mathcal{C}^{*} \\
z^{T}(q+M z) & =0 .
\end{aligned}
$$

We define solvability and feasibility as in Definition II.3. If $\mathcal{C}=\mathbb{R}_{+}^{m}$ then $\operatorname{LCP}_{\mathcal{C}}(q, M)$ becomes the ordinary LCP defined in Definition II.3. The following theorem can be proven by following the footsteps of the proof of Theorem II.4 [10].

Theorem II.7: Let $\mathcal{C}=\mathbb{R}_{+}^{m} \times \mathbb{R}^{\ell}$. Suppose that $M \in \mathbb{R}^{(m+\ell) \times(m+\ell)}$ is nonnegative definite. Then, the following statements are equivalent:

1) $q \in\left(\operatorname{SOL}_{\mathcal{C}}(0, M)\right)^{*}$;

2) $\operatorname{LCP}_{\mathcal{C}}(q, M)$ is feasible;

3) $\operatorname{LCP}_{\mathcal{C}}(q, M)$ is solvable

Moreover, $\operatorname{SOL}_{\mathcal{C}}(q, M)$ is polyhedral and equal to

$$
\begin{aligned}
\left\{z \in \mathbb{R}^{m+\ell} \mid z \in \mathcal{C}, q+M z\right. & \in \mathcal{C}^{*}, q^{T}\left(z-z^{\prime}\right) \\
& \left.=0,\left(M+M^{T}\right)\left(z-z^{\prime}\right)=0\right\}
\end{aligned}
$$

where $z^{\prime}$ is an arbitrary solution of $\operatorname{LCP}_{\mathcal{C}}(q, M)$.

\section{LINEAR NETWORK MODELS}

Consider a linear $k$-port electrical network consisting of only resistors $(R)$, inductors $(L)$, capacitors $(C)$, gyrators $(G)$, and transformers $(T)$. Under suitable conditions (the network does not contain all-capacitor/voltage sources loops or nodes with the only elements incident being inductors/current sources, see [1, 
ch. 4] for more details), this $R L C G T$ circuit can be described by the state-space model

$$
\begin{aligned}
\dot{x}(t) & =A x(t)+B u(t) \\
y(t) & =C x(t)+D u(t)
\end{aligned}
$$

where $A \in \mathbb{R}^{n \times n}, B \in \mathbb{R}^{n \times k}, C \in \mathbb{R}^{k \times n}$, and $D \in \mathbb{R}^{k \times k}$ denote real matrices of appropriate dimensions, and $x$ denotes the state variable of the network (typically consisting of linear combinations of the fluxes through the inductors and charges at the capacitors). The pair $\left(u_{i}, y_{i}\right)$ denotes the voltage-current variables at the ports of the circuit.

Since (3) is a model for an RLCGT-multiport network, the matrix quadruple $(A, B, C, D)$ is not arbitrary, but has a certain structure. Indeed, the system matrices satisfy a property called passivity, which is well known in circuit theory.

Definition III.1 [33]: A system ( $A, B, C, D)$ given by (3) is called passive, or dissipative with respect to the supply rate $u^{T} y$, if there exists a nonnegative-valued function $V: \mathbb{R}^{n} \rightarrow \mathbb{R}_{+}$, (a storage function), such that for all $t_{0} \leqslant t_{1}$ and all time functions $(u, x, y) \in \mathcal{L}_{2}^{k+n+k}\left(t_{0}, t_{1}\right)$ satisfying (3) the following inequality holds:

$$
V\left(x\left(t_{0}\right)\right)+\int_{t_{0}}^{t_{1}} u^{T}(t) y(t) d t \geqslant V\left(x\left(t_{1}\right)\right) .
$$

The above inequality is called the dissipation inequality. The storage function represents a notion of "stored energy" in the network.

Proposition III.2 [33]: Consider a system $(A, B, C, D)$ in which $(A, B, C)$ is a minimal representation. The following statements are equivalent.

- $(A, B, C, D)$ is passive.

- The transfer matrix $G(s):=C(s I-A)^{-1} B+D$ is positive real, i.e., $x^{*}\left[G(\lambda)+G^{*}(\lambda)\right] x \geqslant 0$ for all complex vectors $x$ and all $\lambda \in \mathbb{C}$ such that $\operatorname{Re} \lambda>0$ and $\lambda$ is not an eigenvalue of $A$.

- The matrix inequalities

$$
\left(\begin{array}{cc}
-A^{T} K-K A & -K B+C^{T} \\
-B^{T} K+C & D+D^{T}
\end{array}\right) \geqslant 0
$$

and $K=K^{T} \geqslant 0$ have a solution $K$.

Moreover, in case $(A, B, C, D)$ is passive, all solutions to the linear matrix inequalities (5) are positive definite and a symmetric $K$ is a solution to (5) if and only if $V(x)=1 / 2 x^{T} K x$ defines a storage function of the system $(A, B, C, D)$.

An assumption that we will often use is the following.

Assumption III.3: The matrix $\operatorname{col}\left(B, D+D^{T}\right)$ has full column rank and the triple $(A, B, C)$ is a minimal representation.

These assumptions imply that (specific kinds of) redundancy have been removed from the circuit (see [15] for a discussion).

We note the following consequence of passivity.

Lemma III.4 [15, Lemma III.4] : Consider a system $(A, B, C, D)$ in which $(A, B, C)$ is a minimal representation and $(A, B, C, D)$ is passive. If $v \in \mathbb{R}^{k}$ satisfies $\left(D+D^{T}\right) v=0$ (or equivalently, $v^{T} D v=0$ ), then $C^{T} v=K B v$ for any $K$ satisfying (5).

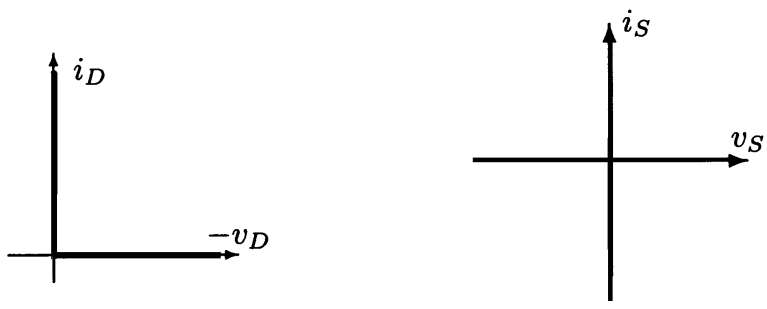

Fig. 1. Voltage-current characteristic of an ideal diode and an ideal switch.

\section{Switched Network Models}

In Section III, we concentrated on linear networks of the form (3). Adding the switches, diodes, and sources will lead to the class of circuits that form the object of study of the paper.

\section{A. Adding Diodes, Switches, and Sources}

The equations that are added to (3) if the terminals are terminated by diodes, switches and sources are given as follows.

- If the $i$ th port is connected to a diode

$$
\left(u_{i}=-V_{i} \wedge y_{i}=I_{i}\right) \vee\left(u_{i}=I_{i} \wedge y_{i}=-V_{i}\right)
$$

where $V_{i}$ and $I_{i}$ are the voltage across and current through the $i$ th diode, respectively, and $\vee$ denotes the Boolean "or" and $\wedge$, the Boolean "and"-operator. The ideal diode characteristics are described by the relations

$$
V_{i} \leqslant 0 \wedge I_{i} \geqslant 0 \wedge\left(V_{i}=0 \vee I_{i}=0\right)
$$

as shown in Fig. 1. Putting the above equations together leads to $0 \leqslant u_{i} \perp y_{i} \geqslant 0$ where $u_{i} \perp y_{i}$ means that the product $u_{i} y_{i}$ is zero or stated otherwise, that either $u_{i}=0$ or $y_{i}=0$.

- If the $i$ th port is connected to a switch

$$
u_{i}=0 \wedge y_{i}=0
$$

or stated differently, $u_{i} \perp y_{i}$ as shown in Fig. 1 .

- If the $i$ th port is connected to a source: $u_{i}$ is actually being described by a suitable function of time, which reflects the applied voltage or current related to the port.

For the sake of brevity, we exclude voltage-current sources in the sequel. All of our results are still valid in the presence of external sources with slight modifications (see [7] for the detailed discussion).

Based on the previous discussion, we obtain network models of the form

$$
\begin{aligned}
\dot{x}(t) & =A x(t)+B u(t) \quad y(t)=C x(t)+D u(t) \\
0 & \leqslant y_{i}(t) \perp u_{i}(t) \geqslant 0, \quad i=1,2, \ldots, m \\
y_{i}(t) & \perp u_{i}(t), \quad i=m+1, m+2, \ldots, k
\end{aligned}
$$

where we assumed that the first $m$ ports are terminated with diodes and the last $k-m$ ports by pure switches. The variable $t \in \mathbb{R}_{+}$denotes time, $x(t)$ the state, and $u(t)$ and $y(t)$ the switch variables at time $t$.

System (7) without the switch conditions (7c) is called a linear complementarity system (LCS). System descriptions of this form were introduced in [28] and were further studied 
in [6], [15]-[17], [29]. Systems without complementarity conditions (7b) have been studied in [13] under a Hamiltonian structure and were called switched Hamiltonian systems (SHS). This paper provides a unified framework that has LCS and SHS as special cases and therefore encompasses a large class of switching circuits. We will use the terminology switched-complementarity systems (SCS) for systems of the form (7) together with the notation $\operatorname{SCS}(A, B, C, D)$.

\section{B. Cone-Complementarity Systems}

A certain similarity between diodes and switches can be made apparent by using a formulation in terms of cones. The constitutive equations for a $k$-tuple of diodes may be written in the form

$$
u \in \mathcal{C} \quad y \in \mathcal{C}^{*} \quad y \perp u
$$

where $\mathcal{C}$ denotes the nonnegative cone $\mathbb{R}_{+}^{k}$ in $\mathbb{R}^{k}$, i.e., the set of $k$ vectors with nonnegative entries. The conditions (8) however become the specification of a set of switches in a particular configuration if we let $\mathcal{C}$ denote a set of the form $\Pi_{i=1}^{k} \mathcal{C}_{i}$ where each $\mathcal{C}_{i}$ is either $\mathbb{R}$ or $\{0\}$. This set is a subspace and so, in particular, it is a cone. The cones corresponding to diodes and to switches may be taken together in a product cone. Consequently, linear RLCTG networks with diodes and switches can always be written in the form

$$
\begin{gathered}
\dot{x}(t)=A x(t)+B u(t) \\
y(t)=C x(t)+D u(t) \\
\mathcal{C}_{\pi(t)}^{*} \ni y(t) \perp u(t) \in \mathcal{C}_{\pi(t)}
\end{gathered}
$$

where $\pi(\cdot)$ is a switching sequence taking values in a finite set $\left\{\pi_{1}, \ldots, \pi_{N}\right\}$, and for each $i$ the set $\mathcal{C}_{\pi_{i}}$ is a closed convex cone in $\mathbb{R}^{k}$.

Early work on cone-complementarity systems in the context of unilaterally constrained systems can be found in [24].

\section{Dynamics in a Given Mode}

Note that (7b) and (7c) imply that for all $i \in\{1,2, \ldots, k\}$ either $y_{i}(t)=0$ or $u_{i}(t)=0$ must be satisfied. In other words, each diode is either conducting or blocking, and each switch is either open or closed. Accordingly, diodes and switches can be replaced by a short or an open circuit.

This results in a multimodal system with $2^{k}$ modes, where each mode is characterized by a subset $\mathcal{I}$ of $\{1,2, \ldots, k\}$, indicating that $y_{i}(t)=0$ if $i \in \mathcal{I}$ and $u_{i}(t)=0$ if $i \in \mathcal{I}^{c}$ with $\mathcal{I}^{c}:=\{1,2, \ldots, k\} \backslash \mathcal{I}$. We split $\mathcal{I}$ as $\mathcal{I}=\mathcal{D} \cup \mathcal{S}$ with $\mathcal{D} \subseteq\{1,2, \ldots, m\}$ and $\mathcal{S} \subseteq\{m+1, m+2, \ldots, k\}$, where $\mathcal{D}$ denotes the status of the diodes and $\mathcal{S}$ of the switches. ${ }^{1}$

For each such mode (also called "topology," "configuration," or "discrete state") the laws of motion are given by differential and algebraic equations (DAEs). Specifically, in mode $\mathcal{I}$ they are given by

$$
\begin{aligned}
\dot{x}(t) & =A x(t)+B u(t) \quad y(t)=C x(t)+D u(t) \\
u_{i}(t) & =0, i \in \mathcal{I}^{c} \quad y_{i}(t)=0, i \in \mathcal{I} .
\end{aligned}
$$

${ }^{1}$ In the sequel of the paper, when we write $\mathcal{D}$ or $\mathcal{S}$, we always mean a subset of $\{1,2, \ldots, m\}$ or $\{m+1, m+2, \ldots, k\}$, respectively. By $\mathcal{D}^{c}$ and $\mathcal{S}^{c}$, we will denote the sets $\{1,2, \ldots, m\} \backslash \mathcal{D}$ and $\{m+1, m+2, \ldots, k\} \backslash \mathcal{S}$, respectively.
During the time evolution of the system, the mode will vary whenever some of the diodes and/or switches change their state (i.e., diodes go from conducting to blocking or vice versa and/or switches from open to close or vice versa). The switch can be considered as time events since an external device triggers the mode change, while the mode transition of the diodes are due to state events: the current mode remains active as long as the inequality conditions in (7b) are satisfied. If they tend to be violated (e.g., the current through the diode tends to become negative) a mode transition occurs.

\section{SOLUTION CONCEPT}

The time evolution of SCS is a sequence of smooth continuations followed by mode transitions.

During the smooth continuations, system trajectories satisfy the DAEs (10) for some mode $\mathcal{I}$ in the classical sense. Hence, it suffices to consider the so-called Bohl functions (see [14]). More precisely, a function $f$ is called a Bohl function (or Bohl type) if $f(t)=H e^{F t} G$ for some matrices $F, G$, and $H$ of appropriate sizes. We denote the set of all Bohl function by $\mathcal{B}$.

At the event of a mode transition, the system may in principle display jumps in the state variable $x$. Jumping phenomena are well-known in the theory of unilaterally constrained mechanical systems [4], where at impacts the change of velocity of the colliding bodies is often modeled as being instantaneous. These discontinuous and impulsive motions are also observed in electrical networks (see, e.g., [11], [22], [25]-[27], [31], [32]).

To obtain a mathematically precise solution concept, we will use a distributional framework. In particular, the Dirac distribution and its derivatives will play a key role.

Definition VI.1: A Bohl distribution is a distribution u of the form $\mathrm{u}=\mathrm{u}_{\mathrm{imp}}+\mathrm{u}_{\mathrm{reg}}$, where

- $\mathrm{u}_{\mathrm{imp}}$ is a linear combination of $\delta$ and its derivatives, i.e., $\mathrm{u}_{\mathrm{imp}}=\sum_{i=0}^{l} u^{-i} \delta^{(i)}$ for real numbers $u^{-i}, i=0, \ldots, l$ and

- $\mathrm{u}_{\mathrm{reg}}$ is a Bohl function on $[0, \infty)$.

The class of Bohl distributions is denoted by $\mathcal{B}_{\text {imp }}$. For a distribution $u \in \mathcal{B}_{\text {imp }}$, $u_{\text {imp }}$ is called the impulsive part and $u_{\text {reg }}$ is called the regular or smooth part. In case $u_{i m p}=0$ we call $u$ a regular or smooth distribution.

Note that the Laplace transform of a Bohl distribution is a rational function. It can be easily verified that a Bohl distribution is regular if and only if its Laplace transform is strictly proper. In what follows, Bohl distributions having a proper Laplace transform will play an important role. We call them first order Bohl distributions. Note that a Bohl distribution is of first order if and only if its impulsive part does not contain the derivatives of Dirac distribution.

With this machinery we can now introduce the concept of an initial solution given an initial state $x(0)=x_{0}$ and a switch configuration $\mathcal{S}$ for the pure switches. This actually implies that $u(t)$ is contained in the cone

$$
\mathcal{C}_{\mathcal{S}}=\left\{v \mid v_{i} \geqslant 0, i=1, \ldots, m, \text { and } v_{i}=0, i \in \mathcal{S}^{c}\right\}
$$

and $y(t)$ should be in the dual cone $\mathcal{C}_{\mathcal{S}}^{*}$. Note that

$$
\mathcal{C}_{\mathcal{S}}^{*}=\left\{v \mid v_{i} \geqslant 0, i=1, \ldots, m, \text { and } v_{i}=0, i \in \mathcal{S}\right\} .
$$


Hence, that means that given $\mathcal{S}$ the governing (7) are reduced to

$$
\begin{aligned}
\dot{x}(t)=A x(t)+B u(t) \quad y(t) & =C x(t)+D u(t) \\
\mathcal{C}_{\mathcal{S}} & \ni u(t) \perp y(t) \in \mathcal{C}_{\mathcal{S}}^{*} .
\end{aligned}
$$

Note that this system can be considered as an extension of the standard LCS in [17] as it used general positive cones $\mathcal{C}_{\mathcal{S}}$. The problem(13) becomes an ordinary LCS when $\mathcal{C}_{\mathcal{S}}=\mathbb{R}_{+}^{k}$.

Note that the "modes" $\mathcal{D}$ of the diodes are not specified by the formulation (13), i.e., $\mathcal{I}=\mathcal{D} \cup \mathcal{S}$ in (10) is not completely known. Hence, a solution in a mode $\mathcal{I}$ being governed by (10) is valid as long as $\mathcal{D}$ does not change. This means that mode $\mathcal{I}$ will only be valid for a limited amount of time in general, since a change of mode (diode going from conducting to blocking or vice versa) may be triggered by the inequality constraints. Therefore, we would like to express some kind of "local satisfaction of the constraints."

We call a (smooth) Bohl function $v$ initially in the cone $\mathcal{C}$ if there exists an $\varepsilon>0$ such that $v(t) \in \mathcal{C}$ for all $t \in[0, \varepsilon)$. We know from the initial value theorem (see, e.g., [12]) that there is a connection between small time values of time functions and large values of the indeterminate $s$ in the Laplace transform. In fact, one can show that $v$ is initially in the cone $\mathcal{C}$ if and only if there exists a $\sigma_{0} \in \mathbb{R}$ such that its Laplace transform $\hat{v}(\sigma) \in \mathcal{C}$ for all $\sigma \geqslant \sigma_{0}$.

The definition of being initially in the cone $\mathcal{C}$ for Bohl distributions will be based on this observation (see also [16]).

Definition VI.2: We call a Bohl distribution v initially in the cone $\mathcal{C}$ if its Laplace transform $\hat{\mathrm{v}}(s)$ satisfies $\hat{\mathrm{v}}(\sigma) \in \mathcal{C}$ for all sufficiently large real $\sigma$.

Remark VI.3: To relate the definition to the time domain, note that a scalar-valued ${ }^{2}$ first-order Bohl distribution $v$ (i.e., $\mathrm{v}_{\mathrm{imp}}=v^{0} \delta$ for some $v^{0} \in \mathbb{R}$ ) is initially in the cone $\mathcal{C}$ if and only if:

1) $v^{0} \in \mathcal{C}$ or

2) $v^{0}=0$ and there exists an $\varepsilon>0$ such that $v_{\text {reg }}(t) \in \mathcal{C}$ for all $t \in[0, \varepsilon)$.

Now, we are in a position to define a local solution concept.

Definition VI.4: We call a Bohl distribution $(\mathrm{u}, \mathrm{x}, \mathrm{y}) \in$ $\mathcal{B}_{\mathrm{imp}}^{k+n+k}$ an initial solution to (7) with initial state $x_{0}$ and pure switch configuration $\mathcal{S}$ if:

1) there is a diode configuration $\mathcal{D}$ such that $(\mathrm{u}, \mathrm{x}, \mathrm{y})$ satisfies (10) for mode $\mathcal{I}=\mathcal{D} \cup \mathcal{S}$ and initial state $x_{0}$ in the distributional sense, i.e., satisfies

$$
\begin{aligned}
\dot{\mathrm{x}}=A \mathrm{x}+B \mathrm{u}+x_{0} \delta & \mathrm{y}=C \mathrm{x}+D \mathrm{u} \\
\mathrm{u}_{i}=0, i \notin \mathcal{I} & \mathrm{y}_{i}=0, i \in \mathcal{I}
\end{aligned}
$$

as equalities of distributions;

2) the pair $(\mathrm{u}, \mathrm{y})$ is initially in the cone $\left(\mathcal{C}_{\mathcal{S}} \times \mathcal{C}_{\mathcal{S}}^{*}\right)$.

Note that condition 2), together with real analyticity of Bohl functions, already implies that (14b) hold for $i \in \mathcal{S}$ and $i \in \mathcal{S}^{*}$, respectively.

For examples of initial solutions in networks without pure switches one can consider [15, Example V.4 , V.5].

\footnotetext{
${ }^{2}$ In this case, the cone $\mathcal{C}$ can only be equal to $\mathbb{R}, \mathbb{R}_{+},-\mathbb{R}_{+}$or $\{0\}$.
}

Theorem VI.5: Consider an SCS given by (7) such that Assumption III.3 is satisfied and $(A, B, C, D)$ represents a passive system. Let a pure switch configuration $\mathcal{S}$ be given and let $\mathcal{Q}_{\mathcal{S}}$ be the solution set of $\operatorname{LCP}_{\mathcal{C}_{\mathcal{S}}}(0, D)$, i.e., $\mathcal{Q}_{\mathcal{S}}=\left\{v \in \mathbb{R}^{k} \mid v \in \mathcal{C}^{\mathcal{S}}, D v \in \mathcal{C}_{\mathcal{S}}^{*}\right.$ and $\left.v \perp D V\right\}$. Then, the following statements hold.

1) For each initial state $x_{0}$, there exists exactly one initial solution to SCS.

2) This solution is of first order. Stated differently, its impulsive part is of the form $\left(u^{0} \delta, 0, D u^{0} \delta\right)$ for some $u^{0} \in \mathcal{Q}_{\mathcal{S}}$.

3) This impulsive part results in a reinitialization (jump) -if applicable- of the state from $x_{0}$ to $x_{0}+B u^{0}$.

4) For all $x_{0} \in \mathbb{R}^{n}, C\left(x_{0}+B u^{0}\right) \in \mathcal{Q}_{\mathcal{S}}^{*}$.

5) The initial solution is smooth (i.e., $u^{0}=0$ ) if and only if $C x_{0} \in \mathcal{Q}_{\mathcal{S}}^{*}$.

Proof:

1) If $\mathcal{S}=\emptyset$, the proof follows from [5, Th. 6.1]. In the general case, we will employ the ideas and techniques that are used in this reference. Define $\mathcal{M}:=\{1,2, \ldots, m\}, q(s):=C(s I-A)^{-1} x_{0}$, and $G(s):=D+C(s I-A)^{-1} B$. Further, define $q^{\prime}(s)=q_{\mathcal{M}}(s)-G_{\mathcal{M S}}(s) G_{\mathcal{S} \mathcal{S}}^{-1}(s) q_{\mathcal{S}}(s)$ and $G^{\prime}(s)=G_{\mathcal{M M}}(s)-G_{\mathcal{M S}}(s) G_{\mathcal{S} \mathcal{S}}^{-1}(s) G_{\mathcal{S M}}(s)$. Now, consider the following complementarity problem:

$$
\begin{aligned}
& \hat{\mathrm{y}}_{\mathcal{M}}(s)=q^{\prime}(s)+G^{\prime}(s) \hat{\mathrm{u}}_{\mathcal{M}}(s) \\
& \hat{\mathrm{u}}_{\mathcal{M}}(\sigma) \geqslant 0 \quad \text { and } \\
& \hat{\mathrm{y}}_{\mathcal{M}}(\sigma) \geqslant 0, \quad \text { for all sufficiently large real } \sigma \\
& \hat{\mathrm{u}}_{\mathcal{M}}(s) \perp \hat{\mathrm{y}}_{\mathcal{M}}(s), \quad \text { for all } s \in \mathbb{C} .
\end{aligned}
$$

Problems of this type are called rational complementarity problems (RCPs). The RCP has been introduced in [28] and further studied in [16]. It is already well-known that there is a one-to-one correspondence between the initial solutions of LCSs and the solutions of RCPs (see [16]). We first suppose that the RCP has a solution $\left(\hat{\mathrm{u}}_{\mathcal{M}}(s), \hat{\mathrm{y}}_{\mathcal{M}}(s)\right)$. Define $\hat{\mathrm{u}}_{\mathcal{S}}(s):=-G_{\mathcal{S} \mathcal{S}}^{-1}(s)\left[q_{\mathcal{S}}(s)+G_{\mathcal{S} \mathcal{M}}(s) \hat{\mathrm{u}}_{\mathcal{M}}(s)\right]$, $\hat{\mathrm{u}}_{\mathcal{S}^{c}}(s):=0, \hat{\mathrm{x}}(s)=(s I-A)^{-1} x_{0}+(s I-A)^{-1} B \hat{\mathrm{u}}(s)$, and finally $\hat{\mathrm{y}}_{\mathcal{S}}(s)=C_{\mathcal{S}^{c} \bullet} \hat{\mathrm{x}}(s)+D_{\mathcal{S}^{c} \bullet \hat{\mathrm{u}}}(s)$. Now, we claim that the inverse Laplace transform of the triple $(\hat{\mathrm{u}}(s), \hat{\mathrm{x}}(s), \hat{\mathrm{y}}(s))$, say $(\mathrm{u}, \mathrm{x}, \mathrm{y})$, is an initial solution to SCS (7) with the initial state $x_{0}$ and pure switch configuration $\mathcal{S}$. Indeed, one can verify that $(\mathrm{u}, \mathrm{x}, \mathrm{y})$ satisfies all the requirements of Definition VI.4 for the diode configuration $\mathcal{D}:=\left\{i \in \mathcal{M} \mid \mathrm{y}_{i}(s) \equiv 0\right\}$. So far, we proved existence of an initial solution provided that the RCP has a solution. Note that $G^{\prime}(s)$ is the Schur complement of $G(s)$ with respect to $G_{\mathcal{S S}}(s)$. It follows from [5, Lemma 3.2, (v)] that $G(\sigma)$ and hence $G^{\prime}(\sigma)$ are positive definite for all sufficiently large real $\sigma$. This implies, together with Theorem II.4 item 1, Remark II.5 and [16, Th. 4.1 and Th. 4.9], that the RCP has a unique solution. At this point, we already showed the existence. Suppose now there are two different initial solutions. Their Laplace transforms should satisfy the relations of the RCP. However, we know that it has a unique solution. 
Note that $\left(\mathrm{u}_{\mathcal{M}}, \mathrm{y}_{\mathcal{M}}\right)$ determines u uniquely since $G_{\mathcal{S S}}(s)$ is invertible due to [5, Lemma 3.2, (v)]. This concludes the uniqueness proof.

2) Let $(u, x, y)$ be the unique initial solution with the Laplace transform $(\hat{\mathrm{u}}(s), \hat{\mathrm{x}}(s), \hat{\mathrm{y}}(s))$. Define $\mathcal{D}:=\{i \in \mathcal{M}$ $\left.\mathrm{y}_{i}(s) \equiv 0\right\}$, and $\mathcal{I}:=\mathcal{D} \cup \mathcal{S}$. The (14) yield $0=$ $C_{\mathcal{I} \bullet}(s I-A)^{-1} x_{0}+G_{\mathcal{I} \mathcal{I}}(s) \hat{\mathrm{u}}_{\mathcal{I}}(s)$ in the Laplace domain. Note that the first summand of the right hand side is strictly proper, $G_{\mathcal{I I}}(s)$ is invertible as a rational matrix (due to [5, Lemma 3.2, (v)]), and $s^{-1} G_{\mathcal{I} \mathcal{I}}^{-1}(s)$ is proper (due to [5, Lemma 3.2, (vi)]). Consequently, $\hat{\mathrm{u}}_{\mathcal{I}}(s)=$ $-G_{\mathcal{I} \mathcal{I}}^{-1}(s) C_{\mathcal{I} \bullet}(s I-A)^{-1} x_{0}$ is proper. We can conclude from (14a) that both $\hat{\mathrm{x}}(s)$ and $\hat{\mathrm{y}}(s)$ are also proper. Therefore, $(\mathrm{u}, \mathrm{x}, \mathrm{y})$ is of first order. Let the impulsive part of $\mathrm{u}$ be of the form $u^{0} \delta$ for some $u^{0} \in \mathbb{R}^{k}$. It is clear from (14a) that $\mathrm{x}$ has no impulsive part and $D u^{0} \delta$ is the impulsive part of y. Note that $u^{0} \in \mathcal{C}_{\mathcal{S}}$ and $D u^{0} \in \mathcal{C}_{\mathcal{S}}^{*}$ due to Definition VI.4 item 2 , and $u^{0}$ is orthogonal to $D u^{0}$ due to (14b). Therefore, $u^{0}$ solves $\operatorname{LCP}_{\mathcal{C}_{\mathcal{S}}}(0, D)$. Theorem II.7 implies that $u^{0} \in \mathcal{Q}_{\mathcal{S}}$.

3) Immediately follows from (14a).

4) Note that (14b) implies that $\hat{\mathrm{u}}^{T}(s) \hat{\mathrm{y}}(s)=0$ for all values of $s \in \mathbb{C}$. Take any $v \in \mathcal{Q}_{\mathcal{S}}$. Then, for all sufficiently large $\sigma$ we have $(\hat{\mathrm{u}}(\sigma)-v)^{T}(\hat{\mathrm{y}}(\sigma)-D v)=-v^{T} \hat{\mathrm{y}}(\sigma)-$ $\hat{\mathrm{u}}^{T}(\sigma) D v \leqslant 0$ since $(\hat{\mathrm{u}}(\sigma), \hat{\mathrm{y}}(\sigma)),(v, D v) \in \mathcal{C}_{\mathcal{S}} \times \mathcal{C}_{\mathcal{S}}^{*}$, and they are pairwise orthogonal. Substituting $\hat{\mathrm{y}}(s)=$ $C(s I-A)^{-1} x_{0}+\left[D+C(s I-A)^{-1} B\right] \hat{\mathrm{u}}(s)$, we get

$$
\begin{aligned}
(\hat{\mathrm{u}}(\sigma)-v)^{T}\left[C(\sigma I-A)^{-1} x_{0}+C(\sigma I-A)^{-1} B \hat{\mathrm{u}}(\sigma)\right. & \\
& +D(\hat{\mathrm{u}}(\sigma)-v)] \leqslant 0
\end{aligned}
$$

for all sufficiently large $\sigma$. Since $D$ is nonnegative definite due to the hypotheses (see [5, Lemma 3.2 (i)]), we have even $(\hat{\mathrm{u}}(\sigma)-v)^{T}\left[C(\sigma I-A)^{-1} x_{0}+C(\sigma I-\right.$ $\left.A)^{-1} B \hat{\mathrm{u}}(\sigma)\right] \leqslant 0$. Multiplying this relation by $\sigma$ and letting $\sigma$ tend to infinity yields

$$
\left(u^{0}-v\right)^{T}\left(C x_{0}+C B u^{0}\right) \leqslant 0 .
$$

Now, let the series expansion of $\hat{\mathrm{u}}(s)$ around infinity be $\hat{\mathrm{u}}(s)=u^{0}+s^{-1} u^{-1}+\cdots$. Hence, we get

$$
\begin{aligned}
\hat{\mathrm{u}}^{T}(s) \hat{\mathrm{y}}(s)= & \left(u^{0}\right)^{T} D u^{0}+s^{-1}\left[\left(u^{0}\right)^{T}\right. \\
& \left.\cdot\left(C x_{0}+C B u^{0}+\left(D+D^{T}\right) u^{-1}\right)\right]+\cdots .
\end{aligned}
$$

Note that $\left(u^{0}\right)^{T} D u^{0}=0$ as proven in 2 . Since $D$ is nonnegative definite due to the hypotheses, we have even $\left(D+D^{T}\right) u^{0}=0$. This means that (16) implies

$$
\left(u^{0}\right)^{T}\left(C x_{0}+C B u^{0}\right)=0 .
$$

Together with (15), this results in $v^{T}\left(C x_{0}+C B u^{0}\right) \geqslant 0$. Since $v \in \mathcal{Q}_{\mathcal{S}}$ is arbitrary, we get $C x_{0}+C B u^{0} \in \mathcal{Q}_{\mathcal{S}}^{*}$.

5) The "only if" part follows from 4. If $C x_{0} \in \mathcal{Q}_{\mathcal{S}}^{*}$ then we get $\left(u^{0}\right)^{T} C x_{0} \geqslant 0$ since $u^{0} \in \mathcal{Q}_{\mathcal{S}}$ as shown in 2. From the proof of the previous item, we already know $\left(u^{0}\right)^{T} D u^{0}=0$ and $\left(u^{0}\right)^{T}\left(C x_{0}+C B u^{0}\right)=0$. This implies from [8, Lemma 20] that $\left(u^{0}\right)^{T} C x_{0}=-\left(u^{0}\right)^{T} C B u^{0} \leqslant 0$. Hence, we get
$\left(u^{0}\right)^{T} C x_{0}=0$ and hence $\left(u^{0}\right)^{T} C B u^{0}=0$. Finally, [8, Lemma 20] gives $u^{0}=0$.

The fact that solutions of linear passive networks with ideal diodes and pure switches do not contain derivatives of Dirac impulses is widely believed true on "intuitive" grounds, but the authors are not aware of any previous rigorous proof. The framework proposed here makes it possible to prove the intuition. Only for the diode case it was proven in [15].

A direct implication of the statements 3,4 , and 5 in Theorem VI.5 is that if smooth continuation is not possible for $x_{0}$, it is possible after one reinitialization. Indeed, by 3 the state after the reinitialization is equal to $x_{0}+B u^{0}$ where $u^{0}$ as in 2 . Since $C\left(x_{0}+B u^{0}\right) \in \mathcal{Q}_{\mathcal{S}}^{*}$ due to 4 , it follows from statement 5 that from $x_{0}+B u^{0}$ there exists a smooth initial solution. This immediately implies local existence (on a time interval $[0$, $\epsilon])$ of a solution.

In [16] and [17] a (global) solution concept for LCS has been introduced that is based on concatenation of initial solutions. In principle, this allows impulses at any mode transition time (necessary for, e.g., unilaterally constrained mechanical systems). However, it has been shown in [15] that such a general notion of solution will not be needed in the context of linear passive electrical networks with diodes.

At this point, we need to introduce some nomenclature. The function space $\mathcal{L}_{\delta}(0, T)$ consists of the distributions of the form $\mathrm{u}=\mathrm{u}_{\mathrm{imp}}+\mathrm{u}_{\mathrm{reg}}$, where $\mathrm{u}_{\mathrm{imp}}=u^{0} \delta$ with $u^{0} \in \mathbb{R}$ and $\mathrm{u}_{\mathrm{reg}} \in$ $\mathcal{L}_{2}(0, T)$.

The following theorem shows the existence and uniqueness of solutions to SCS for a fixed switch configuration.

Theorem VI.6: Consider an SCS given by (7) such that Assumption III.3 is satisfied and $(A, B, C, D)$ represents a passive system. Let a pure switch configuration $\mathcal{S}$ be given. For all initial states $x_{0}$ and all $T>0$, there exists a unique triple $(\mathrm{u}, \mathrm{x}, \mathrm{y}) \in \mathcal{L}_{\delta}^{k+n+k}(0, T)$ such that the following hold.

1) There exists an initial solution $(\overline{\mathrm{u}}, \overline{\mathrm{x}}, \overline{\mathrm{y}})$ such that

$$
\left(\mathrm{u}_{\mathrm{imp}}, \mathrm{x}_{\mathrm{imp}}, \mathrm{y}_{\mathrm{imp}}\right)=\left(\overline{\mathrm{u}}_{\mathrm{imp}}, \overline{\mathrm{x}}_{\mathrm{imp}}, \overline{\mathrm{y}}_{\mathrm{imp}}\right) .
$$

2) $\mathrm{x}_{\mathrm{reg}}(0+)=x_{0}+B u^{0}$ with $u^{0} \in \mathbb{R}^{k}$ given by $\overline{\mathrm{u}}_{\text {imp }}=$ $u^{0} \delta$.

3) For almost all $t \in(0, T)$

$$
\begin{aligned}
\mathrm{x}_{\mathrm{reg}}(t) & =\mathrm{x}_{\mathrm{reg}}(0+)+\int_{0}^{t}\left[A \mathrm{x}_{\mathrm{reg}}(\tau)+B \mathrm{u}_{\mathrm{reg}}(\tau)\right] d \tau \\
\mathrm{y}_{\mathrm{reg}}(t) & =C \mathrm{x}_{\mathrm{reg}}(t)+D \mathrm{u}_{\mathrm{reg}}(t) \\
\mathcal{C}_{\mathcal{S}} & \ni \mathrm{u}_{\mathrm{reg}}(t) \perp \mathrm{y}_{\mathrm{reg}}(t) \in \mathcal{C}_{\mathcal{S}}^{*} .
\end{aligned}
$$

Proof: Since the set of initial states that lead to a smooth initial solution (i.e., $\left\{x_{0} \mid C x_{0} \in \mathcal{Q}_{\mathcal{S}}^{*}\right\}$ ) is a closed set, one can follow the same line of argumentation of the proof of $[15, \mathrm{Th}$. VII.2] step by step.

So far, we were interested in the behavior of SCSs for a fixed switch configuration $\mathcal{S}$. Our next step is to allow changes in switch configuration. To do so, we first describe the allowed switching sequences.

Definition VI.7: A function $\pi: \mathbb{R}_{+} \rightarrow 2^{\{m+1, m+2, \ldots, k\}}$ is said to be an admissible switching function if it is piecewise constant and it changes value at most finitely many times on 
every finite-length interval. The set of point at which $\pi$ changes value will be denoted by $\Gamma_{\pi}$.

Note that $\Gamma_{\pi}$ is set of isolated points due to the fact that there are finitely many points at which $\pi$ changes value on every interval of finite length. By considering only admissible switching sequences, we exclude the so-called Zeno behavior. ${ }^{3}$

As we showed earlier jumps may occur only at switching instants. In what follows, we will adopt a global solution concept which allows jumps at isolated points in time. First, the definition of the trajectory set that we consider is in order.

Definition VI.8: The distribution space $\mathcal{L}_{2, \delta}$ is defined as the set of all $\mathrm{u}=\mathrm{u}_{\mathrm{imp}}+\mathrm{u}_{\mathrm{reg}}$, where $\mathrm{u}_{\mathrm{imp}}=\sum_{\theta \in \Gamma} u^{\theta} \delta_{\theta}$ for $u^{\theta} \in \mathbb{R}$ with $\Gamma \subset \mathbb{R}_{+}$a set of isolated points, and $\mathrm{u}_{\mathrm{reg}} \in \mathcal{L}_{2}^{\text {loc }}$.

The isolatedness of the points of the set $\Gamma$ is required to prevent the occurrence of an accumulation of Dirac impulses in the solution trajectories. One could very well relax this requirement by making some extra assumptions. However, we prefer to keep the definition simpler and avoid technical details which might blur the main picture.

Definition VI.9: Let the impulsive part of the distribution $(\mathrm{u}, \mathrm{x}, \mathrm{y}) \in \mathcal{L}_{2, \delta}^{m+n+m}$ be supported on a set of isolated points $\Gamma$, i.e., $\left(\mathrm{u}_{\mathrm{imp}}, \mathrm{x}_{\mathrm{imp}}, \mathrm{y}_{\mathrm{imp}}\right)=\sum_{\theta \in \Gamma}\left(u^{\theta}, x^{\theta}, y^{\theta}\right) \delta_{\theta}$ for $\left(u^{\theta}, x^{\theta}, y^{\theta}\right) \in \mathbb{R}^{k+n+k}$. Then, we call (u, $\left.\mathrm{x}, \mathrm{y}\right)$ a (global) solution to SCS (7) for the initial state $x_{0}$ and the admissible switching function $\pi$ if the following properties hold.

1) For any interval $(a, b)$ such that $(a, b) \cap \Gamma=\emptyset$ the restriction $\left.\mathrm{x}_{\mathrm{reg}}\right|_{(a, b)}$ is absolutely continuous and satisfies for almost all $t \in(a, b)$

$$
\begin{aligned}
\dot{\mathrm{x}}_{\mathrm{reg}}(t) & =A \mathrm{x}_{\mathrm{reg}}(t)+B \mathrm{u}_{\mathrm{reg}}(t) \\
\mathrm{y}_{\mathrm{reg}}(t) & =C \mathrm{x}_{\mathrm{reg}}(t)+D \mathrm{u}_{\mathrm{reg}}(t) \\
\mathcal{C}_{\pi(t)} & \ni \mathrm{u}_{\mathrm{reg}}(t) \perp \mathrm{y}_{\mathrm{reg}}(t) \in \mathcal{C}_{\pi(t)}^{*} .
\end{aligned}
$$

2) For each $\theta \in \Gamma$ the corresponding impulse $\left(u^{\theta} \delta_{\theta}, x^{\theta} \delta_{\theta}, y^{\theta} \delta_{\theta}\right)$ is equal to the impulsive part of the unique initial solution to (7) with initial state $\mathrm{x}_{\mathrm{reg}}(\theta-):=\lim _{t \uparrow \theta} \mathrm{x}_{\mathrm{reg}}(t)$ (taken equal to $x_{0}$ for $\theta=0$ ).

3) For times $\theta \in \Gamma$ it holds that $\mathrm{x}_{\mathrm{reg}}(\theta+)=\mathrm{x}_{\mathrm{reg}}(\theta-)+$ $B u^{\theta}$.

Note that the solution in the above sense satisfies the equations $\dot{\mathrm{x}}=A \mathrm{x}+B \mathrm{u}$ and $\mathrm{y}=C \mathrm{x}+D \mathrm{u}$ in the distributional sense.

The following theorem establishes existence and uniqueness of solutions to SCS.

Theorem VI.10: Consider an SCS given by (7) such that Assumption III.3 is satisfied and $(A, B, C, D)$ represents a passive system. The SCS (7) has a unique (global) solution (u, $\mathrm{x}, \mathrm{y}) \in$ $\mathcal{L}_{2, \delta}^{m+n+m}$ for any initial state $x_{0}$ and admissible switching function $\pi$. Moreover, $\mathrm{x}_{\mathrm{imp}}=0$ and impulses in $(\mathrm{u}, \mathrm{y})$ only show up at the initial time and times for which $\pi$ changes value (i.e., $\Gamma$ in Definition VI.9 should be a subset of $\{0\} \cup \Gamma_{\pi}$ ).

Proof: A global solution for the switching function $\pi$ can be easily constructed by using Theorem VI.6 repeatedly. For the uniqueness proof, let $\left(\mathrm{u}^{\prime}, \mathrm{x}^{\prime}, \mathrm{y}^{\prime}\right)$ and $\left(\mathrm{u}^{\prime \prime}, \mathrm{x}^{\prime \prime}, \mathrm{y}^{\prime \prime}\right)$ be two dif-

\footnotetext{
${ }^{3}$ The term "Zeno behavior" refers to the phenomenon of an infinite number of events (mode transitions) in a finite-length time interval.
}

ferent global solutions of SCS (7) for the initial state $x_{0}$ and the switching function $\pi$. Let $T \in \Gamma_{\pi}$ be such that

$$
\begin{aligned}
\left.\left(\mathrm{u}^{\prime}, \mathrm{x}^{\prime}, \mathrm{y}^{\prime}\right)\right|_{[0, T)} & =\left.\left(\mathrm{u}^{\prime \prime}, \mathrm{x}^{\prime \prime}, \mathrm{y}^{\prime \prime}\right)\right|_{[0, T)} \\
\left.\left(\mathrm{u}^{\prime}, \mathrm{x}^{\prime}, \mathrm{y}^{\prime}\right)\right|_{[T, \Delta)} & \neq\left.\left(\mathrm{u}^{\prime \prime}, \mathrm{x}^{\prime \prime}, \mathrm{y}^{\prime \prime}\right)\right|_{[T, \Delta)}
\end{aligned}
$$

for some $\Delta$ with $[T, \Delta) \cap \Gamma_{\pi}=\emptyset$. It follows from [15, Th. VII.2] that both $\lim _{t \uparrow T} \mathrm{x}^{\prime}(t)$ and $\lim _{t \uparrow T} \mathrm{x}^{\prime \prime}(t)$ are well defined. Moreover, (18) implies that they are equal. Uniqueness of initial solutions for a given initial state (Theorem VI.5 item 1), together with Definition VI.9 item 2, implies that the impulsive parts of both solutions are the same at $t=T$. Hence, (19) results in

$$
\left.\left(\mathrm{u}_{\text {reg }}^{\prime}, \mathrm{x}_{\mathrm{reg}}^{\prime}, \mathrm{y}_{\text {reg }}^{\prime}\right)\right|_{[T, \Delta)} \neq\left.\left(\mathrm{u}_{\text {reg }}^{\prime \prime}, \mathrm{x}_{\mathrm{reg}}^{\prime \prime}, \mathrm{y}_{\text {reg }}^{\prime \prime}\right)\right|_{[T, \Delta)} .
$$

Note that $\mathrm{x}_{\mathrm{reg}}^{\prime}(T)=\mathrm{x}_{\mathrm{reg}}^{\prime \prime}(T)$. This means that

$$
\left.\left(\mathrm{u}_{\mathrm{reg}}^{\prime}(t)-\mathrm{u}_{\mathrm{reg}}^{\prime \prime}(t), \mathrm{x}_{\mathrm{reg}}^{\prime}(t)-\mathrm{x}_{\mathrm{reg}}^{\prime \prime}(t), \mathrm{y}_{\mathrm{reg}}^{\prime}(t)-\mathrm{y}_{\mathrm{reg}}^{\prime \prime}(t)\right)\right|_{[T, \Delta)}
$$

is a trajectory of the linear system (3) with zero initial state. By using the dissipation inequality, we get

$$
\begin{aligned}
\int_{T}^{t}\left[\mathrm{u}_{\mathrm{reg}}^{\prime}(s)-\mathrm{u}_{\mathrm{reg}}^{\prime \prime}(s)\right]^{T}\left[\mathrm{y}_{\mathrm{reg}}^{\prime}(s)-\mathrm{y}_{\mathrm{reg}}^{\prime \prime}(s)\right] d s \\
\geqslant\left[\mathrm{x}_{\mathrm{reg}}^{\prime}(t)-\mathrm{x}_{\mathrm{reg}}^{\prime \prime}(t)\right]^{T} K\left[\mathrm{x}_{\mathrm{reg}}^{\prime}(t)-\mathrm{x}_{\mathrm{reg}}^{\prime \prime}(t)\right] .
\end{aligned}
$$

Definition VI.9 item 1 implies that the left-hand side is nonpositive. However, the right-hand side is nonnegative due to the fact that $K$ is positive definite. Therefore, $\mathrm{x}_{\mathrm{reg}}^{\prime}(t)=\mathrm{x}_{\mathrm{reg}}^{\prime \prime}(t)$ for all $t \in[T, \Delta)$. This immediately results in

$$
\begin{aligned}
B\left[\mathrm{u}_{\mathrm{reg}}^{\prime}(t)-\mathrm{u}_{\mathrm{reg}}^{\prime \prime}(t)\right] & =0 \\
\mathrm{y}_{\mathrm{reg}}^{\prime}(t)-\mathrm{y}_{\mathrm{reg}}^{\prime \prime}(t) & =D\left[\mathrm{u}_{\mathrm{reg}}^{\prime}(t)-\mathrm{u}_{\mathrm{reg}}^{\prime \prime}(t)\right]
\end{aligned}
$$

due to Definition VI.9. Premultiplying (22) by $\left[\mathrm{u}_{\mathrm{reg}}^{\prime}(t)-\right.$ $\left.u_{\text {reg }}^{\prime \prime}(t)\right]^{T}$, one can show that

$$
\left[\mathrm{u}_{\mathrm{reg}}^{\prime}(t)-\mathrm{u}_{\mathrm{reg}}^{\prime \prime}(t)\right]^{T} D\left[\mathrm{u}_{\mathrm{reg}}^{\prime}(t)-\mathrm{u}_{\mathrm{reg}}^{\prime \prime}(t)\right]=0 .
$$

Since $D$ is nonnegative definite, this implies

$$
\left(D+D^{T}\right)\left[\mathrm{u}_{\mathrm{reg}}^{\prime}(t)-\mathrm{u}_{\mathrm{reg}}^{\prime \prime}(t)\right]=0 .
$$

We can conclude from Assumption III.3, (21), and (23) that $\mathrm{u}_{\mathrm{reg}}^{\prime}(t)-\mathrm{u}_{\mathrm{reg}}^{\prime \prime}(t)=0$ for all $t \in[T, \Delta)$. Finally, (22) gives $\mathrm{y}_{\text {reg }}^{\prime}(t)-\mathrm{y}_{\text {reg }}^{\prime \prime}(t)=0$ for all $t \in[T, \Delta)$. As a consequence, we reached a contradiction with (19).

\section{RegUlar States}

Another consequence of Theorem VI.5 is the characterization of so-called regular states (sometimes also called consistent states) as introduced in the following definition.

Definition VII.1: A state $x_{0}$ is called regular for $\operatorname{SCS}(A, B, C, D)$ with respect to a pure switch configuration if the corresponding initial solution for the same pure switch configuration is smooth. The collection of regular states for a given quadruple $(A, B, C, D)$ with respect to the pure switch configuration $\mathcal{S}$ is denoted by $\mathcal{R}_{\mathcal{S}}$. 
We have the following equivalent characterizations of regular states.

Theorem VII.2: Consider an SCS given by (7) such that Assumption III.3 is satisfied and $(A, B, C, D)$ represents a passive system. Let a pure switch configuration $\mathcal{S}$ be given and let $\mathcal{Q}_{\mathcal{S}}$ be the solution set of $\operatorname{LCP}_{\mathcal{C}_{\mathcal{S}}}(0, D)$, i.e., $\mathcal{Q}_{\mathcal{S}}=\left\{v \in \mathbb{R}^{k} \mid v \in \mathcal{C}_{\mathcal{S}}, D v \in \mathcal{C}_{\mathcal{S}}^{*}\right.$ and $\left.v \perp D v\right\}$. The following statements are equivalent.

1) $x_{0}$ is a regular state for (7) with respect to the pure switch configuration $\mathcal{S}$.

2) $C x_{0} \in \mathcal{Q}_{\mathcal{S}}^{*}$.

3) $\operatorname{LCP}_{\mathcal{C}_{\mathcal{S}}}\left(C x_{0}, D\right)$ has a solution.

4) There exist two vectors $v_{1} \in \mathcal{C}_{\mathcal{S}}^{*}$ and $v_{2} \in \mathcal{C}_{\mathcal{S}}$ such that $C x_{0}=v_{1}-D v_{2} \cdot{ }^{4}$

Proof:

$1 \Leftrightarrow 2$ : This is clear from Theorem VI.5 item 5 .

$2 \Leftrightarrow 3$ : It follows from Theorem II.7.

$3 \Rightarrow 4$ : Note that if $v$ is a solution of $\operatorname{LCP}_{\mathcal{C}_{\mathcal{S}}}\left(C x_{0}, D\right)$ then we can choose $v_{1}:=C x_{0}+D v \in \mathcal{C}_{\mathcal{S}}^{*}$ and $v_{2}:=v \in \mathcal{C}_{\mathcal{S}}$. $4 \Rightarrow 2$ : Let $v_{1} \in \mathcal{C}_{\mathcal{S}}^{*}$ and $v_{2} \in \mathcal{C}_{\mathcal{S}}$ be such that $C x_{0}=$ $v_{1}-D v_{2}$. Take any $w \in \mathcal{Q}_{\mathcal{S}}$. Then, we have

$$
\begin{aligned}
w^{T} C x_{0}= & w^{T} v_{1}-w^{T} D v_{2}=w^{T} v_{1}+(D w)^{T} v_{2} \\
& \left(\text { since } w^{T} D w=0 \text { impliies } D^{T} w=-D w\right) \\
\geqslant & 0\left(\text { since } w \in \mathcal{C}_{\mathcal{S}}, v_{1} \in \mathcal{C}_{\mathcal{S}}^{*}, D w \in \mathcal{C}_{\mathcal{S}}^{*}, \text { and } v_{2} \in \mathcal{C}_{\mathcal{S}}\right) .
\end{aligned}
$$

As a consequence, $C x_{0} \in \mathcal{Q}_{\mathcal{S}}^{*}$.

Hence, several tests are available for deciding the regularity of an initial state $x_{0}$. In [2] it is stated that a well-designed circuit does not exhibit impulsive behavior. As a consequence, the characterization of regular states forms a verification of the synthesis of the network.

In Section VIII, it will be shown that the characterization of the regular states plays a key role in the proof of global existence of solutions as the set of such initial states will be proven to be invariant under the dynamics.

\section{JUMP RULES}

If a state jump occurs at time $t=0$, the new state is given by $x(0+)=x_{0}+B u^{0}$, see Theorem VI.5 item 3 . We now give a characterization of this jump multiplier $u^{0}$ for SCS.

Theorem VIII.1 (Characterization of $u^{0}$ ): Let a switch configuration $\mathcal{S}$ and an initial state $x_{0}$ be given. The following characterizations can be obtained for $u^{0}$.

1) The jump multiplier $u^{0}$ is the unique solution to

$$
\mathcal{Q}_{\mathcal{S}} \ni v \perp C\left(x_{0}+B v\right) \in \mathcal{Q}_{\mathcal{S}}^{*} .
$$

2) The cone $\mathcal{Q}^{*}$ is equal to pos $N:=\{N \lambda \mid \lambda \geqslant 0\}$ and $\mathcal{Q}_{\mathcal{S}}^{*}=\left\{v \mid N^{T} v \geqslant 0\right\}$ for some real matrix $N$. The reinitialized state $\mathrm{x}_{\mathrm{reg}}(0+)$ is equal to $x_{0}+B N \lambda^{0}$ and $u^{0}=N \lambda^{0}$ where $\lambda^{0}$ is a solution of the following ordinary LCP:

$$
0 \leqslant \lambda \perp\left(N^{T} C x_{0}+N^{T} C B N \lambda\right) \geqslant 0 .
$$

${ }^{4}$ When $\mathcal{Q}_{\mathcal{S}}$ is the usual positive cone (i.e., equals to $\mathbb{R}_{+}^{k}$ ), this comes down to saying that $C x_{0}$ is a positive linear combination of the columns of $(I-D)$.
3) The reinitialized state $x_{\text {reg }}(0+)$ is the unique minimum of

$$
\begin{aligned}
& \operatorname{minimize} \frac{1}{2}\left(x-x_{0}\right)^{T} K\left(x-x_{0}\right) \\
& \text { subject to } C x \in \mathcal{Q}_{\mathcal{S}}^{*}
\end{aligned}
$$

and the multiplier $u^{0}$ is uniquely determined by $\mathrm{x}_{\mathrm{reg}}(0+)=x_{0}+B u^{0}$.

4) The jump multiplier $u^{0}$ is the unique minimizer of

$$
\begin{aligned}
& \operatorname{minimize} \frac{1}{2}\left(x_{0}+B v\right)^{T} K\left(x_{0}+B v\right) \\
& \text { subject to } v \in \mathcal{Q}_{\mathcal{S}} .
\end{aligned}
$$

Proof:

1) It is already known from Theorem VI.5 items 2) and 4) that

$$
\begin{aligned}
u^{0} & \in \mathcal{Q}^{*} \\
C x_{0}+C B u^{0} & \in \mathcal{Q}_{\mathcal{S}}^{*} .
\end{aligned}
$$

Furthermore, (17) readily shows

$$
u^{0} \perp C\left(x_{0}+B u^{0}\right) .
$$

It remains to prove that $u^{0}$ is uniquely determined by (24). Suppose that $z^{i}$ is a solution of the generalized LCP

$$
\begin{aligned}
z & \in \mathcal{Q}^{*} \\
C x_{0}+C B z & \in \mathcal{Q}_{\mathcal{S}}^{*} \\
z^{T}\left(C x_{0}+C B z\right) & =0
\end{aligned}
$$

for $i=1,2$. Note that

$$
\begin{aligned}
& \left(z^{1}-z^{2}\right)^{T} C B\left(z^{1}-z^{2}\right) \\
= & \left(z^{1}-z^{2}\right)^{T}\left[\left(C x_{0}+C B z^{1}\right)-\left(C x_{0}+C B z^{2}\right)\right] \\
= & -\left(z^{1}\right)^{T}\left(C x_{0}+C B z^{2}\right)-\left(z^{2}\right)^{T}\left(C x_{0}+C B z^{1}\right)
\end{aligned}
$$

and hence

$$
\left(z^{1}-z^{2}\right)^{T} C B\left(z^{1}-z^{2}\right) \leqslant 0 .
$$

Since $\mathcal{Q}^{*} \subseteq \operatorname{ker}\left(D+D^{T}\right)$, we have $z^{1}-z^{2} \in$ $\operatorname{ker}\left(D+D^{\bar{T}}\right)$. Hence, $\left(z^{1}-z^{2}\right)^{T} C B\left(z^{1}-z^{2}\right)=$ $\left(z^{1}-z^{2}\right)^{T} B^{T} K B\left(z^{1}-z^{2}\right) \geqslant 0$ due to [5, Lemma 3.2 (iii)]. Together with the above inequality, this gives $\left(z^{1}-z^{2}\right)^{T} C B\left(z^{1}-z^{2}\right)=\left(z^{1}-z^{2}\right)^{T} B^{T} K B\left(z^{1}-z^{2}\right)=$ 0 . Since $\operatorname{col}\left(B, D+D^{T}\right)$ is of full column rank and $K$ is positive definite, we get $z^{1}=z^{2}$. Consequently, the jump multiplier $u^{0}$ is uniquely determined by (24).

2) Since $(A, B, C, D)$ is passive, $D$ is necessarily nonnegative definite. It follows from Theorem II.7 that $\operatorname{SOL}(0, D)$ is a polyhedral cone, i.e., the solution set of a homogeneous system of inequalities of the form $H x \geqslant 0$ for some matrix $H$. Minkowski's theorem [30, Th. 2.8.6] states that every polyhedral cone has a finite set of generators. Therefore, one can find a matrix $N$ such that $\mathcal{Q}_{\mathcal{S}}=\operatorname{pos} N=\{N \lambda \mid \lambda \geqslant 0\}$. It can be checked that the dual cone can be given in the form $\mathcal{Q}_{\mathcal{S}}^{*}=\left\{v \mid N^{T} v \geqslant 0\right\}$. Since $u^{0} \in \mathcal{Q}_{\mathcal{S}}$, there exists $\lambda^{0} \geqslant 0$ such that $u^{0}=N \lambda^{0}$. 
Note that $C x_{0}+C B N \lambda^{0} \in \mathcal{Q}_{\mathcal{S}}^{*}$. Hence, $N^{T}\left(C x_{0}+\right.$ $\left.C B N \lambda^{0}\right) \geqslant 0$. Note that we have

$$
\left(\lambda^{0}\right)^{T} N^{T}\left(C x_{0}+F w(0)+C B N \lambda^{0}\right)=0
$$

due to previous item. This means that $\lambda^{0}$ is a solution of the LCP (25).

3) The minimization problem (26) admits a unique solution since $\left\{x \mid C x \in \mathcal{Q}_{\mathcal{S}}^{*}\right\}$ is a polyhedron and $K$ is positive definite. Let $\bar{x}$ be the solution of (26). Dorn's duality theorem [21, Th. 8.2.4] implies that there exists a $\bar{\lambda}$ such that the pair $(\bar{x}, \bar{\lambda})$ solves

$$
\begin{aligned}
& \operatorname{minimize} x^{T} K x \\
& \text { subject to } \lambda \geqslant 0 \text { and } x=x_{0}+B N \lambda .
\end{aligned}
$$

Since $N \lambda \in \mathcal{Q}_{\mathcal{S}} \subseteq \operatorname{ker}\left(D+D^{T}\right)$ for all $\lambda \geqslant 0$, it follows that $K B N \lambda=C^{T} N \lambda$ for all $\lambda \geqslant 0$ due to Lemma III.4. Thus

$$
\begin{aligned}
x^{T} K x & =\left(x_{0}+B N \lambda\right)^{T} K\left(x_{0}+B N \lambda\right) \\
& =\lambda^{T} N^{T} C B N \lambda+2 x_{0}^{T} C^{T} N \lambda+x_{0}^{T} K x_{0}
\end{aligned}
$$

whenever $\lambda \geqslant 0$. So, the vector $\bar{\lambda}$ solves the minimization problem

$$
\begin{aligned}
& \text { minimize } \frac{1}{2} \lambda^{T} N^{T} C B N \lambda+\left(C x_{0}\right)^{T} N \lambda \\
& \text { subject to } \lambda \geqslant 0 .
\end{aligned}
$$

Since $N^{T} C B N$ is nonnegative definite, the Karush-Kuhn-Tucker conditions

$$
\begin{aligned}
\bar{\lambda} & \geqslant 0 \\
N^{T}\left(C x_{0}+C B N \bar{\lambda}\right) & \geqslant 0 \\
\bar{\lambda}^{T} N^{T}\left(C x_{0}+C B N \bar{\lambda}\right) & =0
\end{aligned}
$$

are necessary and sufficient for the vector $\bar{\lambda}$ to be a globally optimal solution of (34). For a detailed discussion on this equivalence, the reader is referred to [9] or [10, Sec. 1.2]. Note that the LCP given by (35) is the same as the one in (ii). It follows from (ii) that $u^{0}=N \bar{\lambda}$ and $\bar{p}=x_{0}+B u^{0}$. Since $u^{0} \in \operatorname{ker}\left(D+D^{T}\right)$ and $\operatorname{col}\left(B, D+D^{T}\right)$ is of full column rank (due to Assumption III.3), the equation $\mathrm{x}_{\mathrm{reg}}(0+)=x_{0}+B u^{0}$ determines the multiplier $u^{0}$ uniquely.

\section{STABILITY}

In this section, we discuss the stability of SCS under a passivity assumption. The Lyapunov stability of hybrid and switched systems in general has already received considerable attention [3], [18]-[20], [23], [34]. We have narrowed down the definitions and theorems on the stability of general hybrid systems from [19] and [34] to apply to SCS. From now on, we denote the unique global trajectory for a given switch function $\pi$ and initial state $x_{0}$ of an SCS by $\left(\mathrm{u}^{\pi, x_{0}}, \mathrm{x}^{\pi, x_{0}}, \mathrm{y}^{\pi, x_{0}}\right)$. For the study of stability we consider the source-free case.

Definition IX.1 (Equilibrium Point): A state $\bar{x}$ is an equilibrium point of the SCS (7), if for all admissible switching func- tions $\pi \mathrm{x}_{\mathrm{reg}}^{\pi, \bar{x}}(t)=\bar{x}$ for almost all $t \geqslant 0$ and all $\pi$, i.e., for all solutions starting in $\bar{x}$ the state stays in $\bar{x}$.

Note that in an equilibrium point $\dot{x}=0$, which leads in a simple way to the following characterization of equilibria of an SCS.

Lemma IX.2: A state $\bar{x}$ is an equilibrium point of the SCS (7), if and only if for all $\mathcal{S} \subset\{m+1, \ldots, k\}$ there exist $u^{\mathcal{S}} \in \mathbb{R}^{k}$ and $y^{\mathcal{S}} \in \mathbb{R}^{k}$ satisfying

$$
\begin{aligned}
0 & =A \bar{x}+B u^{\mathcal{S}} \\
y^{\mathcal{S}} & =C \bar{x}+D u^{\mathcal{S}} \\
\mathcal{C}_{\mathcal{S}} & \ni u^{\mathcal{S}} \perp y^{\mathcal{S}} \in \mathcal{C}_{\mathcal{S}}^{*} .
\end{aligned}
$$

Moreover, this means that $\bar{x} \in \mathcal{R}_{\mathcal{S}}$ for all $\mathcal{S}$, i.e., $\bar{x}$ is a regular state for all switch configurations.

From this lemma it follows that $\bar{x}=0$ is an equilibrium. Note that if $A$ is invertible we get $\bar{x}=-A^{-1} B u^{\mathcal{S}}$ and

$$
\mathcal{C}_{\mathcal{S}} \ni u^{\mathcal{S}} \perp\left[-C A^{-1} B+D\right] u^{\mathcal{S}} \in \mathcal{C}_{\mathcal{S}}^{*}
$$

which is a homogeneous LCP over a cone.

Definition IX.3: Let $\bar{x}$ be an equilibrium point of the SCS (7) and $d$ denote a metric on $\mathbb{R}^{n}$.

1) $\bar{x}$ is called stable, if for every $\varepsilon>0$ there exists a $\delta>0$ such that $d\left(\mathrm{x}_{\mathrm{reg}}^{\pi, x_{0}}(t), \bar{x}\right)<\varepsilon$ for almost all $t \geqslant 0$ whenever $d\left(x_{0}, \bar{x}\right)<\delta$ and $\pi$ being an admissible switching function.

2) $\bar{x}$ is called asymptotically stable if $\bar{x}$ is stable and there exists $\delta>0$ such that $\lim _{t \rightarrow \infty} d\left(\mathrm{x}_{\mathrm{reg}}^{\pi, x_{0}}(t), \bar{x}\right)=0$ whenever $d\left(x_{0}, \bar{x}\right)<\delta$ and $\pi$ being an admissible switching function. By $\lim _{t \rightarrow \infty} d\left(\mathrm{x}_{\mathrm{reg}}^{\pi, x_{0}}(t), \bar{x}\right)=0$ we mean that for every $\varepsilon>0$ there exists a $t_{\varepsilon}$ such that $\left.d\left(\mathrm{x}_{\mathrm{reg}}^{\pi, x_{0}}(t), \sigma\right), \bar{x}\right)<\varepsilon$ whenever $t \geqslant t_{\varepsilon}$.

In the proof of the main theorem on stability we will need the following lemma.

Lemma IX.4: For a given $\mathcal{S}$ and vectors $w_{1} \in \mathcal{Q}_{\mathcal{S}}=$ $S O L_{\mathcal{C}_{\mathcal{S}}}(0, D)$ and $w_{2} \in \mathcal{Q}_{\mathcal{S}}$, it holds that $w_{1}^{T} D w_{2}=0$.

Proof: Since $D w_{2} \in \mathcal{C}_{\mathcal{S}}^{*}$ and $w_{1} \in \mathcal{C}_{\mathcal{S}}$, it holds that $w_{1}^{T} D w_{2} \geqslant 0$. Note that $w_{2} \in \mathcal{Q}_{\mathcal{S}}$ implies that $w_{2}^{T} D w_{2}=0$ and thus $D w_{2}=-D^{T} w_{2}$. Hence

$$
w_{1}^{T} D w_{2}=-w_{1}^{T} D^{T} w_{2}=-(\underbrace{D w_{1}}_{\in \mathcal{C}_{\mathcal{S}}^{*}})^{T} \underbrace{w_{2}}_{\in \mathcal{C}_{\mathcal{S}}} \leqslant 0
$$

the result follows.

Theorem IX.5: Consider an SCS given by (7) such that Assumption III.3 is satisfied and $(A, B, C, D)$ represents a passive system. This SCS has only stable equilibrium points $\bar{x}$. Moreover, if $A^{T} K+K A<0$ is invertible ${ }^{5} \bar{x}=0$ is the only equilibrium point, which is asymptotically stable.

Proof: Let $\bar{x}$ be an equilibrium. The proof will be based on taking $V(x)=1 / 2(x-\bar{x})^{T} K(x-\bar{x})$ as a Lyapunov function with $K$ a positive definite solution to (5). Take an initial state $x_{0}$ and an admissible switching function $\pi$ and denote the corresponding solution by $\left(\mathrm{u}^{\pi, x_{0}}, \mathrm{x}^{\pi, x_{0}}, \mathrm{y}^{\pi, x_{0}}\right)$. If we apply the same switching function to $\operatorname{SCS}(A, B, C, D)$ with ini-

\footnotetext{
${ }^{5}$ This implies that the linear matrix inequality (5) is strict in the $x$ variable and thus that $A$ is stable. In the case of a Hamiltonian framework this means in the current setting that $R>0$.
} 
tial state $\bar{x}$, then the solution is equal to $t \mapsto\left(u^{\pi(t)}, \bar{x}, y^{\pi(t)}\right)$, where $u^{\pi(t)}$ and $y^{\pi(t)}$ are the vectors that satisfy the conditions in Lemma IX.2. Note that the difference trajectory $\left(\mathrm{u}^{\pi, x_{0}}\right.$ $\left.u^{\pi(\cdot)}, \mathrm{x}^{\pi, x_{0}}-\bar{x}, \mathrm{y}^{\pi, x_{0}}-y^{\pi(\cdot)}\right)$ is a (distributional) solution to the linear system (3). From Definition VI.9, it follows that that jumps of this trajectory only take place at the initial time 0 and the discontinuity points of $\pi$ being $\Gamma_{\pi}$. In intervals between these "jump times," the difference trajectory is smooth and satisfies the dissipation inequality meaning that for $t_{0} \leqslant t_{1}$ (we drop the "reg" subscript as we consider times intervals $\left[t_{0}, t_{1}\right]$ in which no impulses are active)

$$
\begin{aligned}
& \frac{1}{2}\left(\mathrm{x}^{\pi, x_{0}}\left(t_{0}\right)-\bar{x}\right)^{T} K\left(\mathrm{x}^{\pi, x_{0}}\left(t_{0}\right)-\bar{x}\right) \\
& \quad+\int_{t_{0}}^{t_{1}}\left[\mathrm{u}^{\pi, x_{0}}(t)-u^{\pi(t)}\right]^{T}\left[\mathrm{y}^{\pi, x_{0}}(t)-y^{\pi(t)}\right] d t \\
& \quad \geqslant \frac{1}{2}\left(\mathrm{x}^{\pi, x_{0}}\left(t_{1}\right)-\bar{x}\right)^{T} K\left(\mathrm{x}^{\pi, x_{0}}\left(t_{1}\right)-\bar{x}\right) .
\end{aligned}
$$

Since $\mathrm{u}^{\pi, x_{0}}(t) \perp \mathrm{y}^{\pi, x_{0}}(t), u^{\pi(t)} \perp y^{\pi(t)},-\left(u^{\pi(t)}\right)^{T} \mathrm{y}^{\pi, x_{0}}(t) \leqslant$ 0 and $-\left(\mathrm{u}^{\pi, x_{0}}(t)\right)^{T} y^{\pi(t)} \leqslant 0$, it follows that

$$
\begin{aligned}
\frac{1}{2}\left(\mathrm{x}^{\pi, x_{0}}\left(t_{0}\right)-\bar{x}\right)^{T} K\left(\mathrm{x}^{\pi, x_{0}}\left(t_{0}\right)-\bar{x}\right) & \\
& \geqslant \frac{1}{2}\left(\mathrm{x}^{\pi, x_{0}}\left(t_{1}\right)-\bar{x}\right)^{T} K\left(\mathrm{x}^{\pi, x_{0}}\left(t_{1}\right)-\bar{x}\right)
\end{aligned}
$$

for all intervals $\left[t_{0}, t_{1}\right]$ not containing jumps and impulses. Hence, the Lyapunov function cannot increase on these intervals.

The only issue left to prove, to obtain stability according to the standard theorems from [19] and [34], is the fact that the $V(x)$ decreases during jumps of the state trajectory satisfying the equations of $\operatorname{SCS}(A, B, C, D)$. If a jump occurs it obeys the rules as indicated in item 4 in Theorem VIII.1. Let a jump take place from $x_{0}$ (or any other state) and $v$ the corresponding multiplier. As $0 \in \mathcal{Q}_{\mathcal{S}}$ it follows from item 4, that $\left(x_{0}+B v\right)^{T} K\left(x_{0}+B v\right) \leqslant x_{0}^{T} K x_{0}$, or stated differently

$$
v^{T} B^{T} K x_{0}+x_{0}^{T} K B v+v^{T} B^{T} K B v \leqslant 0 .
$$

Consider the difference between the value of the Lyapunov function after and before the jump

$$
\begin{aligned}
2\left(V\left(x_{0}+B v\right)-V\left(x_{0}\right)\right)= & -v^{T} B^{T} K\left(x_{0}-\bar{x}\right) \\
& -\left(x_{0}-\bar{x}\right)^{T} K B v+v^{T} B^{T} K B v .
\end{aligned}
$$

Then, we get

$$
\begin{aligned}
& 2\left(V\left(x_{0}+B v\right)-V\left(x_{0}\right)\right) \\
\leqslant & -v^{T} B^{T} K \bar{x}-\bar{x} K B v[\text { from }(37)] \\
= & \left.-v^{T} C \bar{x}-\bar{x}^{T} C^{T} v \text { [from Lemma III.4 as } v^{T} D v=0\right] \\
= & -2 v^{T}\left[y^{\mathcal{S}}-D u^{\mathcal{S}}\right. \text { ] [due to Lemma IX.2] } \\
\leqslant & 0 \quad\left[\text { Lemma IX.4 and } v^{T} y^{\mathcal{S}} \geqslant 0\right] .
\end{aligned}
$$

This means that during jumps and smooth continuation the Lyapunov function never increases.

Consider the Lyapunov function $V(x)$ for $\bar{x}=0$. It can actually be shown that $d V / d t \leqslant(1 / 2) x^{T}(t)\left[A^{T} K+K A\right] x(t)$ along a solution trajectory, which implies that only the origin is an equilibrium and it is asymptotically stable.

\section{$\mathrm{X}$. Conclusions}

Our aim in this paper has been to demonstrate that a suitable framework for switched piecewise-linear networks is provided by the notion of cone-complementarity systems. The dynamics described by cone-complementarity systems can be very complicated but nevertheless is given by two simple components, to wit a linear system and a closed convex cone. Switching may be described within this context in a conceptually straightforward way as switching between cones, while the underlying linear system remains the same.

Making use of impulsive-smooth distributions to define a sufficiently flexible notion of solution, we have shown that the framework of cone-complementarity systems is sound in the sense that, under the passivity assumption, it produces unique solutions for any given initial state. Moreover, the framework allows formal proofs for intuitive properties concerning jumps and stability. We have obtained a characterization of the situations in which jumps occur as well as of the extent of the jump in these cases; this information should be useful both for theoretical and for simulation purposes.

The cones that we have considered are in fact of a special type in which each component is either unconstrained, constrained to be zero, or constrained to be nonnegative. The formulation of cone-complementarity systems however invites a less coordinate based and more geometric perspective, which helps to achieve a focus on basic issues. Some of the results that we have obtained in this paper still make use of the special properties of cones obtained from diodes and switches; it is a natural question to ask whether these results can be obtained at a more general level, and we intend to return to this in future work.

Another possible direction of generalization is concerned with nonlinear networks. The notion of passivity of course does not depend on linearity and so it seems reasonable to expect that many of the results in this paper can be generalized to the nonlinear case. However, the distributional framework seems less suited in connection with nonlinear dynamics and so a different setting will have to be chosen.

The notion of passivity has been crucial in this paper. In fact, it is remarkable that this energy-related concept turns out to play an important role even in establishing existence and uniqueness of solutions in a context that involves switching.

\section{REFERENCES}

[1] B. D. O. Anderson and S. Vongpanitlerd, Network Analysis and Synthesis. A Modern Systems Theory Approach. Englewood Cliffs, NJ: Pentice-Hall, 1973.

[2] D. Bedrosian and J. Vlach, "Time-domain analysis of networks with internally controlled switches," IEEE Trans. Circuits Syst. I, vol. 39, pp. 199-212, Mar. 1992.

[3] M. S. Branicky, "Multiple Lyapunov functions and other analysis tools for switched and hybrid systems," IEEE Trans. Automat. Contr., vol. 43, pp. 475-482, Apr. 1998.

[4] B. Brogliato, Nonsmooth Impact Mechanics. Models, Dynamics and Control. London, U.K.: Springer, 1996, vol. 220.

[5] M. K. Çamlıbel, W. P. M. H. Heemels, and J. M. Schumacher, "On linear passive complementarity systems," Euro. J. Contr., vol. 8, no. 3, pp. 220-237, 2002. 
[6] M. K. Çamlıbel, M. K. K. Cevik, W. P. M. H. Heemels, and J. M. Schumacher, "From Lipschitzian to non-Lipschitzian characteristics: Continuity of behaviors," in Proc. 39th IEEE Conf. Decision and Control, vol. 5, Sydney, Australia, 2000, pp. 4373-4378.

[7] M. K. Çamlıbel, W. P. M. H. Heemels, A. J. v. d. Schaft, and J. M. Schumacher, "Modeling, well-posedness and stability of switched electrical networks," Eindhoven University of Technology, Dept. Electrical Engineering, Control Systems Group, Eindhoven, The Netherlands, 02I/01, 2002.

[8] M. K. Çamlıbel, W. P. M. H. Heemels, and J. M. Schumacher, "Consistency of a time-stepping method for a class of piecewise-linear networks," IEEE Trans. Circuits Syst. I, vol. 49, pp. 349-357, Mar. 2002.

[9] R. W. Cottle and G. B. Dantzig, "Complementarity pivot theory of mathematical programming," in Studies in Optimization, G. Dantzig and B. Eaves, Eds. Washington, DC: Math. Assoc. Amer., 1974, vol. 10, pp. $27-51$.

[10] R. W. Cottle, J.-S. Pang, and R. E. Stone, The Linear Complementarity Problem. Boston, MA: Academic, 1992.

[11] A. Dervisoglu, "State equations and initial values in active $R L C$ networks," IEEE Trans. Circuit Theory, vol. 18, pp. 544-547, Sept. 1971.

[12] J.J.DiStefano, A. R. Stubberud, and I. J. Williams, Theory and Problems of Feedback and Control Systems. New York: McGraw-Hill, 1967.

[13] K. M. Gerritsen, A. J. van der Schaft, and W. P. M. H. Heemels, "On switched Hamiltonian systems," in Proc. 15th Int. Symp. Mathematical Theory of Networks and Systems (MTNS2002), South Bend, IN, 2002.

[14] M. L. J. Hautus and L. M. Silverman, "System structure and singular control," Linear Algebra Applicat., vol. 50, pp. 369-402, 1983.

[15] W. P. M. H. Heemels, M. K. Çamlıbel, and J. M. Schumacher, "On the dynamic analysis of piecewise-linear networks," IEEE Trans. Circuits Syst. I, vol. 49, pp. 315-327, Mar. 2002.

[16] W. P. M. H. Heemels, J. M. Schumacher, and S. Weiland, "The rational complementarity problem," Linear Algebra Applicat., vol. 294, pp. 93-135, 1999.

[17] — , "Linear complementarity systems," SIAM J. App. Math., vol. 60, no. 4, pp. 1234-1269, 2000.

[18] M. Johansson and A. Rantzer, "Computation of piecewise quadratic Lyapunov functions for hybrid systems," IEEE Trans. Automat. Contr., vol. 43, pp. 555-559, Apr. 1998.

[19] Z. Li, C. B. Soh, and X. Xu, "Lyapunov stability of a class of hybrid dynamic systems," Automatica, vol. 36, no. 2, pp. 297-302, 2000.

[20] D. Liberzon and A. S. Morse, "Basic problems in stability and design of switched systems," IEEE Contr. Syst. Mag., vol. 19, pp. 59-70, May 1999.

[21] O. L. Mangasarian, Nonlinear Programming. New York: McGrawHill, 1969.

[22] A. Massarini, U. Reggiani, and K. Kazimierczuk, "Analysis of networks with ideal switches by state equations," IEEE Trans. Circuits Syst. I, vol. 44, pp. 692-697, Aug. 1997.

[23] A. N. Michel, "Recent trends in the stability analysis of hybrid dynamical systems," IEEE Trans. Circuits Syst. I, vol. 46, pp. 120-134, Jan. 1999.

[24] J. J. Moreau, "Unilateral contact and dry friction in finite freedom dynamics," in CISM Courses and Lecture Notes, J. J. Moreau and P. Panagiotopoulos, Eds. New York: Springer-Verlag, 1988, vol. 302.

[25] Y. Murakami, "A method for the formulation and solution of circuits composed of switches and linear RLC elements," IEEE Trans. Circuits Syst., vol. 34, pp. 496-509, May 1987.

[26] A. Opal and J. Vlach, "Consistent initial conditions of nonlinear networks with switches," IEEE Trans. Circuits Syst.I, vol. 38, pp. 698-710, July 1991.

[27] S. S. Sastry and C. A. Desoer, "Jump behavior of circuits and systems," IEEE Trans. Circuits Syst. I, vol. 28, pp. 1109-1124, Dec. 1981.

[28] A. J.v. d. Schaft and J. M. Schumacher, "The complementary-slackness class of hybrid systems," Math.Contr., Signals Syst., vol.9, pp. 266-301, 1996.

[29] —_ "Complementarity modeling of hybrid systems," IEEE Trans. Automat. Contr., vol. 43, pp. 483-490, Apr. 1998.

[30] J. Stoer and C. Witzgall, Convexity and Optimization in Finite Dimensions I. Berlin, Germany: Springer, 1970.

[31] J. Tolsa and M. Salichs, "Analysis of linear networks with inconsistent initial conditions," IEEE Trans. Circuits Syst. I, vol. 40, pp. 885-894, Dec. 1993.

[32] J. Vlach, J. M. Wojciechowski, and A. Opal, "Analysis of nonlinear networks with inconsistent initial conditions," IEEE Trans. Circuits Syst. I, vol. 42, pp. 195-200, Apr. 1995.
[33] J. C. Willems, "Dissipative dynamical systems," Arch. Rational Mech. Anal., vol. 45, pp. 321-393, 1972 .

[34] H. Ye, A. N. Michel, and L. Hou, "Stability theory for hybrid dynamical systems," IEEE Trans. Automat. Contr., vol. 43, pp.461-474, Apr. 1998.

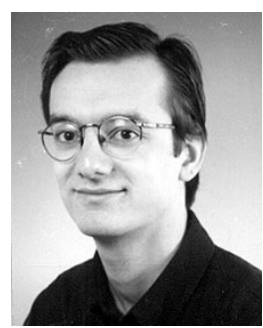

M. K. Çamlıbel was born in Istanbul, Turkey, in 1970. He received the B.Sc. and M.Sc. degrees in control and computer engineering from the Istanbul Technical University, Istanbul, Turkey, in 1991 and 1994, respectively, and the Ph.D. degree from Tilburg University, Tilburg, The Netherlands, in 2001.

He currently holds a part-time Assistant Professor position at Dogus University, Istanbul and a part-time Post-Doctoral position at Tilburg University. His main research interests include the analysis and control of nonsmooth dynamical systems, in particular complementarity systems.

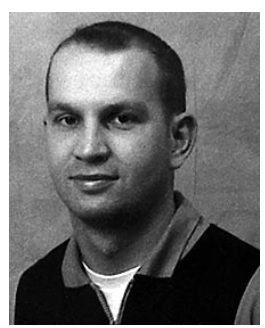

W. P. M. H. Heemels was born in St. Odilienberg, The Netherlands, in 1972. He received the M.Sc. degree (with honors) in mathematics and the Ph.D. degree (cum laude) in electrical engineering, from the Eindhoven University of Technology, Eindhoven, The Netherlands, in 1995 and 1999, respectively.

Currently, he is an Assistant Professor in the Control Systems Group, Department of Electrical Engineering, Eindhoven University of Technology. His research interests include modeling, analysis and control of hybrid systems and dynamics under inequality constraints (especially complementarity problems and systems).

Dr. Heemels was awarded the ASML price for the best Ph.D. dissertation of the Eindhoven University of Technology in 1999/2000 in the area of fundamental research.

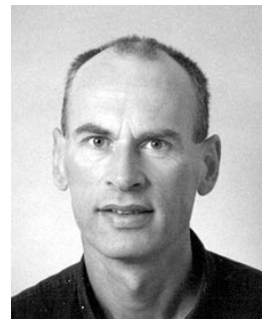

A. J. van der Schaft (M'91-SM'98-F'02) received the Bachelor's and Ph.D. degrees in mathematics from the University of Groningen, Groningen, The Netherlands, in 1979 and 1983, respectively.

In 1982, he joined the Department of Mathematics, University of Twente, Enschede, The Netherlands, where he is presently a Full Professor in Mathematical Systems and Control Theory. His research interests include the mathematical modeling of physical and engineering systems, and the analysis and control of nonlinear and hybrid

systems.

J. M. Schumacher was born in Heemstede, The Netherlands, in 1951. He received the M.Sc. and Ph.D. degrees in mathematics, from the Vrije Universiteit, Amsterdam, The Netherlands, in 1976 and 1981, respectively.

Following postdoctoral positions at the Massachusetts Institute of Technology, Cambridge, MA, the Erasmus University, Rotterdam, The Netherlands, and at European Space Research and Technology Center (ESTEC), Noordwijk, The Netherlands, he was affiliated with the Centre for Mathematics and Computer Science (CWI), Amsterdam, The Netherlands, from 1984 until 1999. $\mathrm{He}$ is now Full Professor of Mathematics in the Department of Econometrics and Operations Research, Tilburg University, Tilburg, The Netherlands. His current research interests are in mathematical finance and nonsmooth dynamical systems.

Dr Schumacher has served as Corresponding Editor of the SIAM Journal on Control and Optimization and is an Associate Editor of Systems and Control Letters 